\title{
Fluctuation of riding passengers induced by chaotic motions of shuttle buses
}

\author{
Takashi Nagatani* \\ Department of Mechanical Engineering, Division of Thermal Science, Shizuoka University, Hamamatsu 432-8561, Japan
}

(Received 23 April 2003; published 12 September 2003)

\begin{abstract}
We study the fluctuation of the number of riding passengers in a few shuttle buses that pass each other freely. We present a dynamical model of the shuttle buses that takes into account the maximum capacity of a bus. The dynamics of the buses is expressed in terms of a coupled nonlinear map with noise. The number of passengers carried by a bus and the time headway between buses exhibit complex behavior with varying trips. It is found that the behavior of the buses exhibits deterministic chaos even if there is no noise. The chaotic motion depends on the loading parameter, the maximum capacity of a bus, the bus's speed, and the number of buses. When the loading parameter is larger than a threshold value, each bus carries a full load of passengers throughout its trip. The dependence of the threshold (transition point) on both capacity and speed is clarified.

DOI: 10.1103/PhysRevE.68.036107

PACS number(s): 89.40.-a, 05.45.-a, 82.40.Bj
\end{abstract}

\section{INTRODUCTION}

Recently, transportation problems have attracted much attention in the field of physics [1-5]. Traffic flow, pedestrian flow, and bus-route problems have been studied from the points of view of statistical mechanics and nonlinear dynamics [6-23]. Interesting dynamical phase transitions have been found in transportation systems. Jams are a typical signature of the complex behavior of traffic flow. Chaos has also been found in traffic flow [24,25].

A shuttle bus system exhibits severe congestion problems in peak traffic. The maximum rate of serving passengers is achieved if each bus carries a full load of passengers throughout its trip. Furthermore, when the number of passengers is larger than the maximum, queuing occurs at the starting point. Queuing is similar to a jam in traffic flow. Below the full load, the number of riding passengers fluctuates greatly. In managing a shuttle bus operation, the usual criterion for deciding the number of buses is that one should be able to transport everyone from starting point to destination within some period of time for rush hour trips [26-28]. Another criterion used in shuttle bus operation is that a passenger's waiting time should not exceed some specified value.

Various models of bus route systems have been studied. In a bus route model with many buses, it has been found that a bunching transition between an inhomogeneous jammed and a homogeneous phase occurs with increasing bus density [17-22]. In a cyclic system where the buses do not pass each other, it has been shown that the buses exhibit such complex behaviors as periodic and chaotic motions [27]. It has also been found that a distinct chaotic motion is induced when buses pass each other freely in a system including a few shuttle buses [28]. However, there are few dynamical models to estimate the dynamic behavior of shuttle buses that take into account the maximum capacity of the buses. It is important and necessary to estimate the number of riding passengers on the buses. Fluctuation of the number of riding passengers will be induced not only by stochastic variation in passengers arriving at the starting point, but also by varying

\footnotetext{
*Email address: tmtnaga@ipc.shizuoka.ac.jp
}

headway between a bus and the bus ahead of it. The difference between the arrival times of buses varies from time to time. Fluctuations of the number of riding passengers may be attributable to the variation between the time headways. It is expected that the capacity of the bus also has an important effect on the fluctuation of riding passenger numbers.

In this paper, we present a simplified dynamical model to describe the motions of buses taking into account the maximum capacity of the buses. We study the dynamical behavior of buses induced by the interaction between buses through the number of passengers waiting at the starting point, when the buses shuttle between the starting point and the destination repeatedly. We investigate the number of passengers carried by the buses and the time headway between the buses. We also study the effect of the bus capacity on the motion and the number of riding passengers.

\section{MODEL}

We consider the dynamical behavior of $M$ buses shuttling between the starting point (origin) and the destination. The waiting passengers at the origin board a bus just arrived, then the bus starts at the origin, moves toward the destination, all currently riding passengers leave the bus when the bus arrives at the destination, and the bus returns to the origin. When a bus reaches other buses, it passes them freely. If the number of waiting passengers is greater than the maximum capacity of the bus, the passengers board the bus to capacity and the remaining passengers wait for the next bus. We assume that the buses keep a constant mean speed on the route.

We define the number of passengers boarding bus $i$ for trip $m$ by $B_{i}(m)$. The parameter $\gamma$ is the time it takes one passenger to board the bus, so $\gamma B_{i}(m)$ is the amount of time needed to board all the passengers at the origin. The parameter $\eta$ is the time it takes one passenger to leave the bus, so $\eta B_{i}(m)$ is the amount of time needed to leave all the passengers at the destination. The moving time of bus $i$ is $2 L / V_{i}$, where $L$ is the distance between the origin and the destination, and $V_{i}$ is the mean speed of bus $i$. The tour time equals the sum of these periods. Then the arrival time $t_{i}(m$ +1 ) of bus $i$ at the origin for trip $m+1$ is given by 


$$
t_{i}(m+1)=t_{i}(m)+(\gamma+\eta) B_{i}(m)+\frac{2 L}{V_{i}}
$$

for $i=1,2, \ldots, M$.

We define $W_{i}(m)$ as the number of passengers waiting at the origin just before bus $i$ arrives at the origin for trip $m$. It is expressed by

$$
W_{i}(m)=W_{i^{\prime}}\left(m^{\prime}\right)-B_{i^{\prime}}\left(m^{\prime}\right)+\mu\left(t_{i}(m)-t_{i^{\prime}}\left(m^{\prime}\right)\right)+\xi_{i}(m),
$$

where bus $i^{\prime}$ is the one arriving at the origin just before bus $i$ and trip $m, t_{i^{\prime}}\left(m^{\prime}\right)$ is the arrival time of bus $i^{\prime}$ at the origin, and $B_{i^{\prime}}\left(m^{\prime}\right)$ is the number of passengers boarding bus $i^{\prime}$. New passengers arrive at the origin at rate $\mu$. So $\mu\left(t_{i}(m)\right.$ $\left.-t_{i^{\prime}}\left(m^{\prime}\right)\right)$ is the number of passengers that have arrived since the bus ahead $\left(i^{\prime}\right)$ left the origin. The number of new passengers fluctuates from time to time. We take into account the fluctuation of new passengers. The fluctuation is represented by white noise $\xi_{i}(m)$ where $\left\langle\xi_{i}(m)\right\rangle=0$ and $\left\langle\xi_{i}(m) \xi_{i^{\prime}}\left(m^{\prime}\right)\right\rangle=a^{2} \delta_{i i^{\prime}} \delta_{m m^{\prime}}$.

We define the maximum capacity of bus $i$ as $F_{i \text {,max }}$. The passenger number $B_{i}(m)$ boarding bus $i$ for trip $m$ is given by

$$
B_{i}(m)=\min \left[F_{i, \max }, W_{i}(m)\right] .
$$

If the number of passengers waiting at the origin is higher than the maximum capacity, the number of passengers boarding the bus is limited by the maximum capacity. The remaining passengers wait for the next bus. Dividing the actual time by the characteristic time $2 L / V_{0}$ ( $V_{0}$ is the reference speed), one obtains the following equation for the dimensionless arrival time of bus $i$ at the origin:

$$
T_{i}(m+1)=T_{i}(m)+\Gamma B_{i}(m)+\frac{V_{0}}{V_{i}},
$$

with

$$
W_{i}(m)=W_{i^{\prime}}\left(m^{\prime}\right)-B_{i^{\prime}}\left(m^{\prime}\right)+\Pi\left(T_{i}(m)-T_{i^{\prime}}\left(m^{\prime}\right)\right)+\xi_{i}(m)
$$

and

$$
B_{i}(m)=\min \left[F_{i, \max }, W_{i}(m)\right],
$$

where $\quad T_{i}(m) \equiv t_{i}(m) V_{0} / 2 L, \quad \Gamma \equiv(\gamma+\eta) V_{0} / 2 L, \quad$ and $\quad \Pi$ $\equiv \mu 2 L / V_{0}$.

Thus, the dynamics of the buses is described by the simplified map (4) with Eqs. (5) and (6). The map should be iterated simultaneously for $M$ buses. The dynamical properties of the map are controlled by four parameters: the loading parameter $\Gamma \Pi$, the capacity $F_{i \text {,max }}$, the bus speed $V_{i} / V_{0}$, and the bus number $M$. When the number of prospective passengers increases, the value of the loading parameter becomes high.

For a few buses, the order of the buses changes at trip $m$ and the time headway between buses changes from trip to trip because a bus passes other buses or is outstripped by
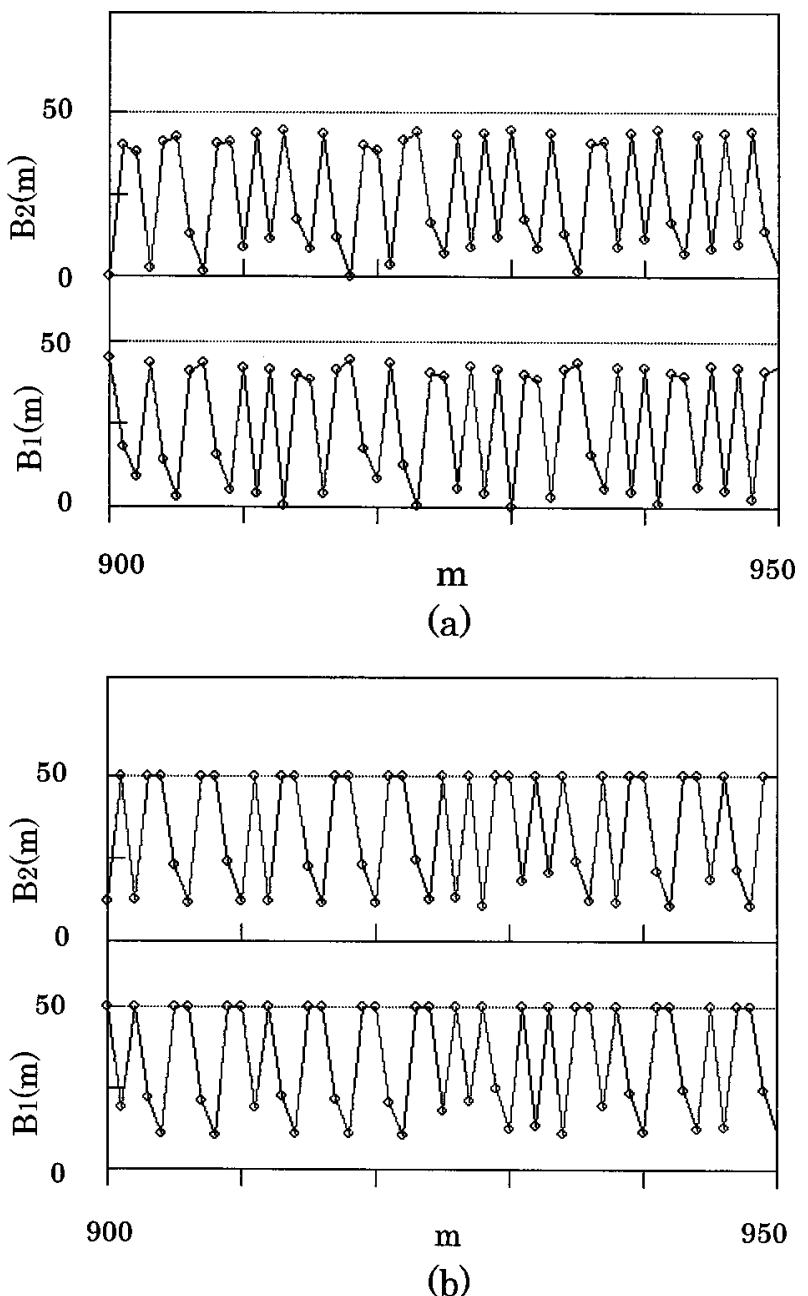

FIG. 1. Plots of the numbers $B_{1}(m)$ and $B_{2}(m)$ of riding passengers on buses 1 and 2 against trip number $m$ from trip $m=900$ to 950 for the values of $\Pi=$ (a) 40 and (b) 50 and no noise, $a=0.0$, where $M=2, \Gamma=0.01$, and $F_{1, \max }=F_{2, \max }=50$. The dotted line indicates the maximum capacity $F_{1 \text { (or } 2 \text { ), max }}$.

other buses. As a result, the number of riding passengers also changes from bus to bus. It is expected that the buses will exhibit a complex behavior.

\section{SIMULATION RESULTS}

We investigate the dynamical behavior of shuttle buses by use of iterates of the map (4)-(6). We calculate the time headway between the buses and the number of riding passengers for various trips. We study the fluctuations of the number of riding passengers and the time headway, the effects of a speed difference and a capacity difference on bus behavior, and the noise effect on bus behavior.

\section{A. Two identical buses}

We investigate the dynamical behavior for two identical buses. Figure 1 shows plots of the numbers $B_{1}(m)$ and $B_{2}(m)$ of riding passengers on buses 1 and 2 against trip number $m$ from trip $m=900$ to 950 for the values of $\Pi$ $=$ (a) 40 and (b) 50 and no noise, $a=0.0$, where $M=2, \Gamma$ 


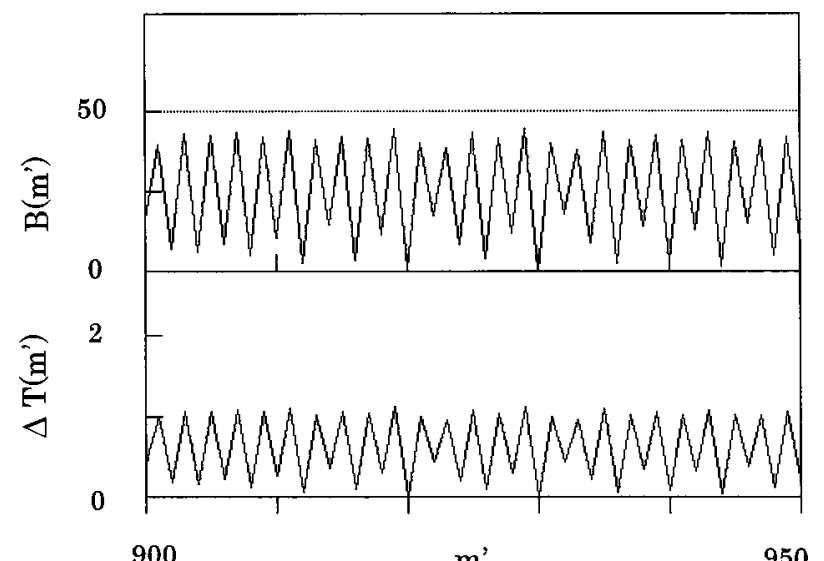

(a)

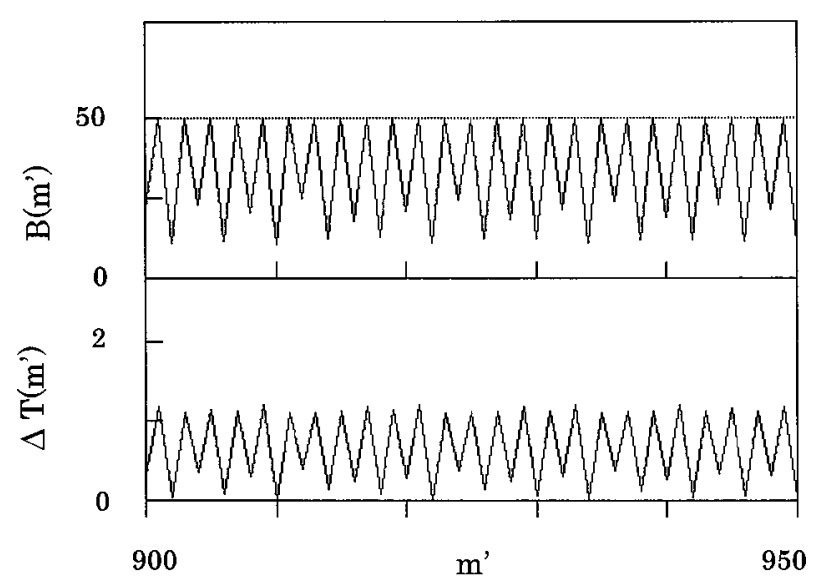

(b)

FIG. 2. Plots of the time headway $\Delta T\left(\mathrm{~m}^{\prime}\right)$ and the number $B\left(m^{\prime}\right)$ of riding passengers against arrival order $m^{\prime}$ from $m^{\prime}$ $=900$ to 950 for the values of $\Pi=$ (a) 40 and (b) 50, where (a) and (b) correspond, respectively, to (a) and (b) in Fig. 1.

$=0.01, V_{1}=V_{2}=V_{0}$, and $F_{1, \max }=F_{2, \max }=50$. The dotted line indicates the maximum capacity $F_{1 \text { (or } 2) \text {,max }}$. The numbers $B_{1}(m)$ and $B_{2}(m)$ of riding passengers cannot be greater than $F_{1 \text { (or 2),max }}$. For case (a) of a low value of loading parameter, the numbers of riding passengers change irregularly from zero to a value lower than the maximum capacity $F_{1 \text { (or 2),max }}$. For case (b), the numbers of riding passengers change irregularly from nonzero to the maximum capacity $F_{1 \text { (or 2),max }}$. The buses frequently carry a full load of passengers.

Figure 2 shows plots of the time headway $\Delta T\left(m^{\prime}\right)$ and the number $B\left(\mathrm{~m}^{\prime}\right)$ of riding passengers against arrival order $m^{\prime}$ from $m^{\prime}=900$ to 950 for the values of $\Pi=$ (a) 40 and (b) 50 where (a) and (b) correspond, respectively, to (a) and (b) in Fig. 1 The time headway changes alternately from low to high values. The number of riding passengers changes similarly by synchronizing with the time headway. Both time headway and number of riding passengers vary almost periodically with period 2 . However, these quantities exhibit an irregularity. When two buses arrive simultaneously at the origin, the time headway becomes zero. As a result, the riding passenger number becomes zero because the number

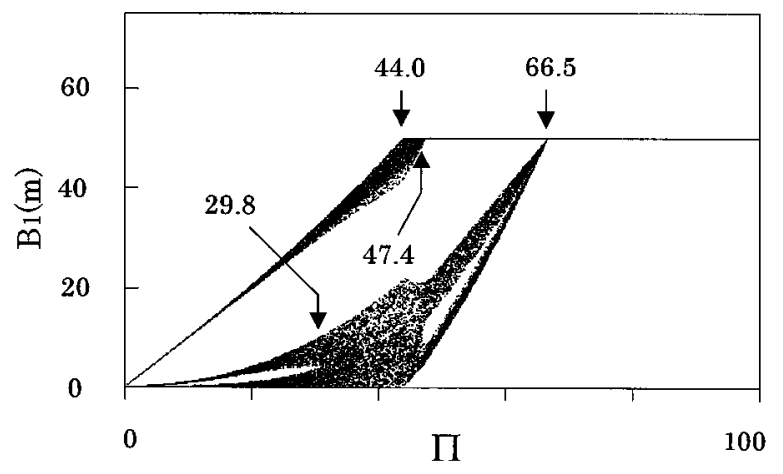

(a)

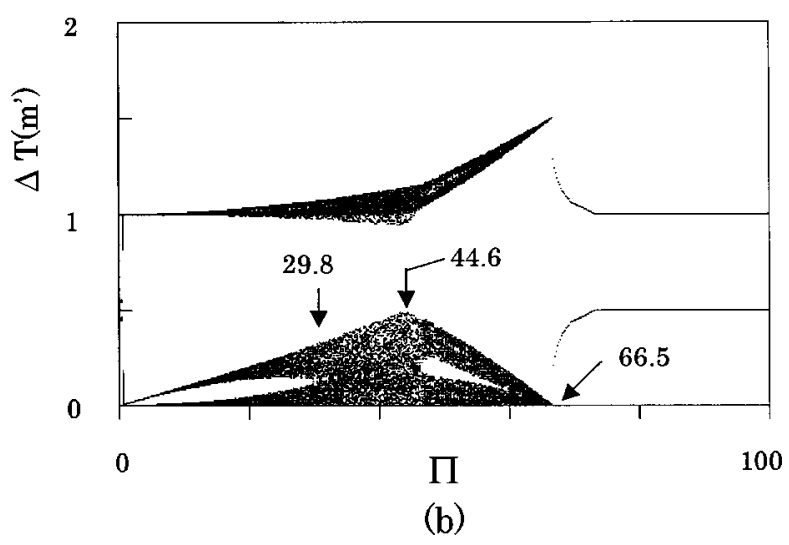

FIG. 3. (a) Plot of the number $B_{1}(m)$ of riding passengers on bus 1 against loading parameter $\Pi$ from sufficiently large trip number $m=900$ to 1000 where $M=2, \Gamma=0.01, F_{1, \max }=F_{2, \max }=50$, and $a=0.0$. (b) Plot of the time headway $\Delta T(m)$ against loading parameter $\Pi$.

of waiting passengers is proportional to the time headway for no queuing at the origin. In (b), even if two buses arrive simultaneously at the origin, the riding passenger number does not become zero when the bus ahead carries the full load of passengers.

We study the distribution of the number of riding passengers and the time headway for various values of the loading parameter in the case of two buses without noise. Figure 3(a) shows a plot of the number $B_{1}(m)$ of riding passengers on bus 1 against loading parameter $\Pi$ from sufficiently large trip number $m=900$ to 1000 where $M=2, \Gamma=0.01, F_{1, \max }$ $=F_{2, \max }=50$, and $a=0.0$. Figure $3(\mathrm{~b})$ shows a plot of the time headway $\Delta T(m)$ against loading parameter $\Pi$ from sufficiently large trip number $m=900$ to 1000 where the values of the parameters are the same as in Fig. 3(a).

For low values of the loading parameter $\Pi$, both the number of riding passengers and the time headway take three values and their distributions exhibit three peaks around these values. With increasing loading parameter $\Pi$, the localized distributions extend around the peaks and become two extended distributions for $29.8<\Pi<47.4$. When the loading parameter $\Pi$ is higher than 47.4 , the two extended distributions break down into three distributions again. If the loading parameter $\Pi$ is higher than 66.5 , the time headway takes two values periodically. For $\Pi<66.5$, the time headway exhibits chaotic motion. Thus, a dynamical transition from chaotic to 
periodic motion occurs at $\Pi_{c}=66.5$.

The distribution of the number of riding passengers exhibits similar behavior to that of the time headway. When the loading parameter is higher than $\Pi=44.0$, the number of riding passengers saturates sometimes at $F_{1 \text { (or 2), max }}$. For $47.4<\Pi<66.5$, either bus 1 or bus 2 carries a full load of passengers. When the loading parameter $\Pi$ is higher than 66.5 , both numbers $B_{1}(m)$ and $B_{2}(m)$ saturate at the maximum capacity. At $\Pi_{c}=66.5$, the two buses are fully loaded. Therefore, the dynamical transition from chaotic to periodic motion at $\Pi_{c}=66.5$ is induced by fully loaded buses.

We study the probability density $f\left(B_{1}\right)$ against passenger number $B_{1}$ for the values of $\Pi=40$ and 50 and no noise, $a$ $=0.0$, where $M=2, \Gamma=0.01$, and $F_{1, \max }=F_{2, \max }=50$. The probability density distributions correspond, respectively, to those in Figs. 1(a) and 1(b). For $\Pi=40$, the probability density exhibits two separate distributions. This means that the number of riding passengers varies greatly from $0<B_{1}<18$ to $37<B_{1}<44$. For $\Pi=50$, the probability density exhibits a distribution with the three peaks. The third peak shows the full load of passengers. Thus, the number of riding passengers varies greatly in chaotic motion.

We study the mean and root mean square of the number $B_{1}(m)$ of riding passengers, where $M=2, \Gamma=0.01, F_{1, \max }$ $=F_{2 \text {, max }}=50$, and $a=0.0$. The mean value increases monotonically according as the loading parameter increases and saturates at the transition point $\Pi_{c}=66.5$. The root mean square increases with loading parameter, reaches its highest value at $\Pi=47.5$, and then decreases and becomes zero at the transition point $\Pi_{c}=66.5$. The value $\Pi=47.5$ at the highest value of the rms corresponds to the point where one bus carries the full load of passengers and the other bus does not.

\section{B. Effect of a speed difference}

We study the effect of a speed difference between two buses on the behavior of the number of riding passengers. We set the mean speeds of two buses as $V_{1}=V_{0}$ and $V_{2}$ $=2 V_{0} / 3$. Bus 2 moves more slowly than bus 1 . Thus the dynamical motion of bus 2 is definitely different from the behavior of bus 1. Figure 4 shows plots of the numbers $B_{1}(m)$ and $B_{2}(m)$ of riding passengers on buses 1 and 2 against trip number $m$ from $m=900$ to 950 for the values of $\Pi=$ (a) 30 and (b) 50 and no noise, $a=0.0$, where $M=2$, $\Gamma=0.01$, and $F_{1, \max }=F_{2, \max }=50$. The dotted line indicates the maximum capacity $F_{1 \text { (or } 2) \text {,max }}$. The numbers $B_{1}(m)$ and $B_{2}(m)$ of riding passengers cannot be greater than $F_{1 \text { (or 2),max }}$. For case (a) of a low value of the loading parameter, the numbers of riding passengers change irregularly from zero to a value lower than the maximum capacity $F_{1 \text { (or 2),max }}$. However, the behavior of the riding passenger number of bus 1 is different from that of bus 2. On average, the riding passenger number of bus 1 is higher than that of bus 2. For case (b), the numbers of riding passengers change irregularly from nonzero to the maximum capacity $F_{1 \text { (or 2),max }}$. The buses frequently carry the full load of passengers. On average, the riding passenger number of bus 1 is

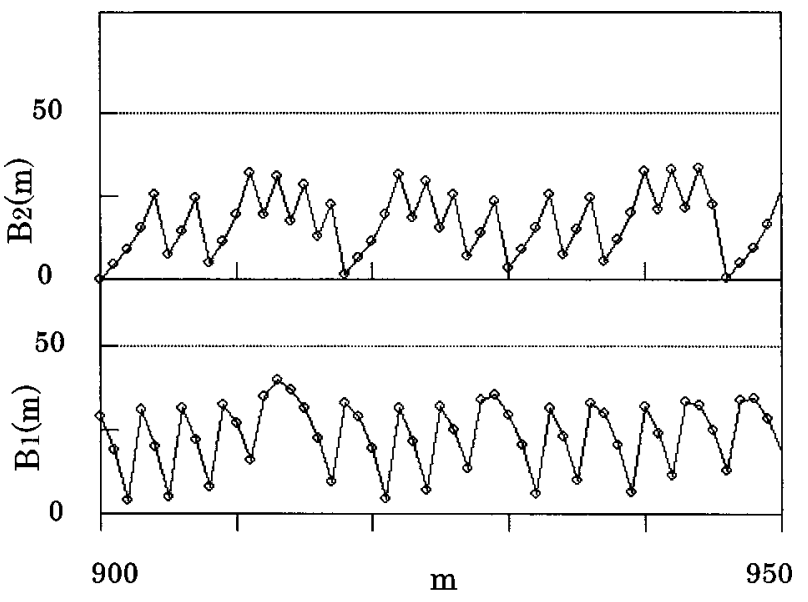

(a)

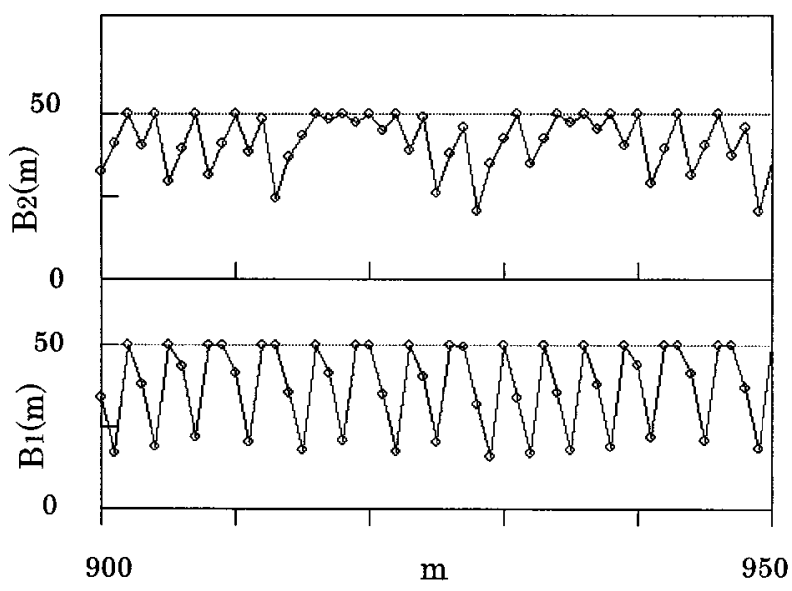

(b)

FIG. 4. Plots of the numbers $B_{1}(m)$ and $B_{2}(m)$ of riding passengers on buses 1 and 2 with different speeds against trip number $m$ from $m=900$ to 950 for the values of $\Pi=$ (a) 30 and (b) 50 and no noise, $a=0.0$, where $M=2, \Gamma=0.01, V_{1}=V_{0}, V_{2}=2 V_{0} / 3$, and $F_{1, \max }=F_{2, \max }=50$.

less than that of bus 2. The dynamical behavior of bus 1 is definitely different from that of bus 2 .

Figure 5(a) shows a plot of the number $B_{1}(\mathrm{~m})$ of riding passengers on bus 1 against loading parameter $\Pi$ from sufficiently large trip number $m=900$ to 1000 where $M=2, \Gamma$ $=0.01, F_{1, \max }=F_{2, \max }=50$, and $a=0.0$. Figure $5(\mathrm{~b})$ shows a plot of the number $B_{2}(m)$ of riding passengers on bus 2 against loading parameter $\Pi$. Both numbers of riding passengers on buses 1 and 2 exhibit extended distributions. The extended distributions are definitely different from those in Fig. 3(a). With increasing loading parameter $\Pi$, the full load of bus 1 begins at $\Pi=35.6$ while the full load of bus 2 begins at $\Pi=40.5$. Bus 1 becomes fully loaded before bus 2 . If the loading parameter is higher than $\Pi=40.0$, both buses are always not empty. When the loading parameter is higher than $\Pi_{c}=58.3$, both buses carry the full load of passengers. Thus, the dynamical transition from chaotic to periodic motion occurs at $\Pi_{c}=58.3$. The transition point is less than $\Pi_{c}=66.5$ in Fig. 3(a). 


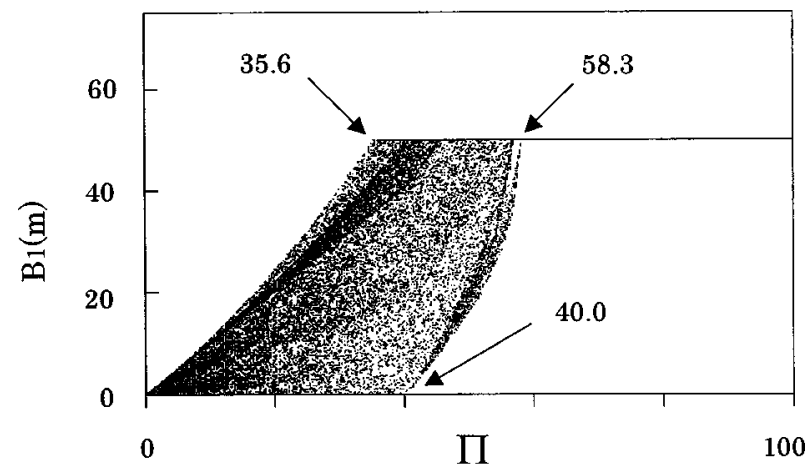

(a)

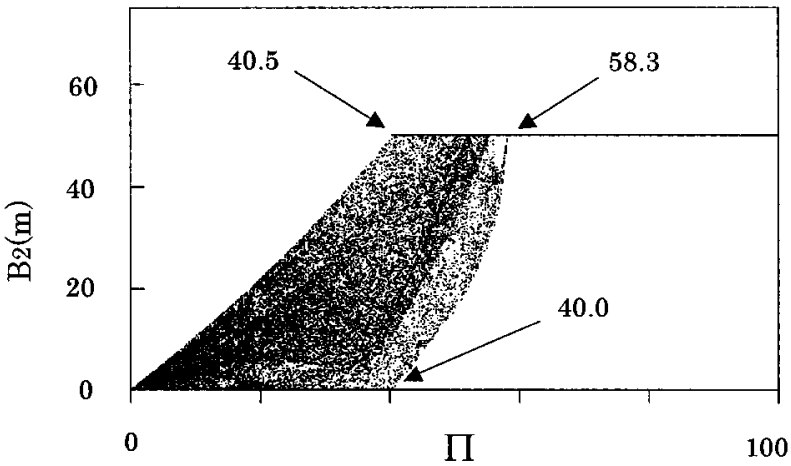

(b)

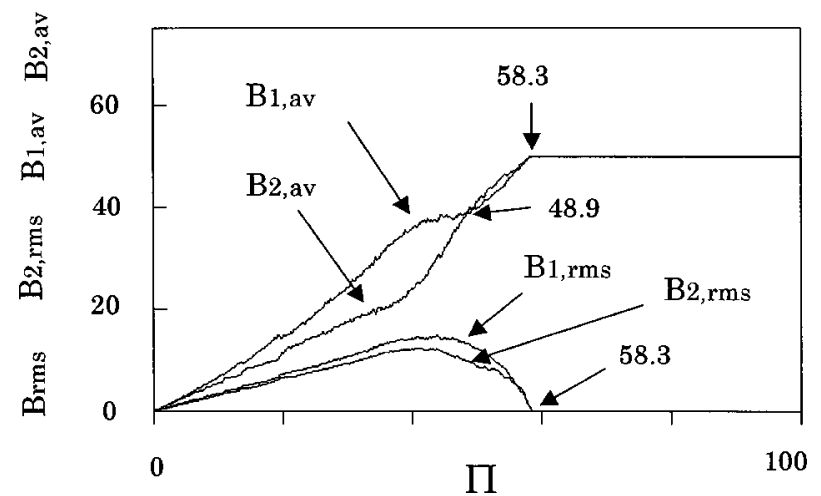

FIG. 6. Plots of means $B_{1, \text { av }}$ and $B_{2, \text { av }}$ and root mean squares $B_{1, \mathrm{rms}}$ and $B_{2, \mathrm{rms}}$ of riding passenger numbers against loading parameter $\Pi$ where $M=2, \Gamma=0.01, V_{1}=V_{0}, V_{2}=2 V_{0} / 3, F_{1, \max }$ $=F_{2, \max }=50$, and $a=0.0$.

and then decrease and become zero at the transition point $\Pi_{c}=58.3$.

\section{Effect of a capacity difference}

We study the effect of a capacity difference between buses on the number of riding passengers. Figure 7(a) shows a plot of number $B_{1}(\mathrm{~m})$ of riding passengers on bus 1 against the loading parameter $\Pi$ from sufficiently large trip number $m$
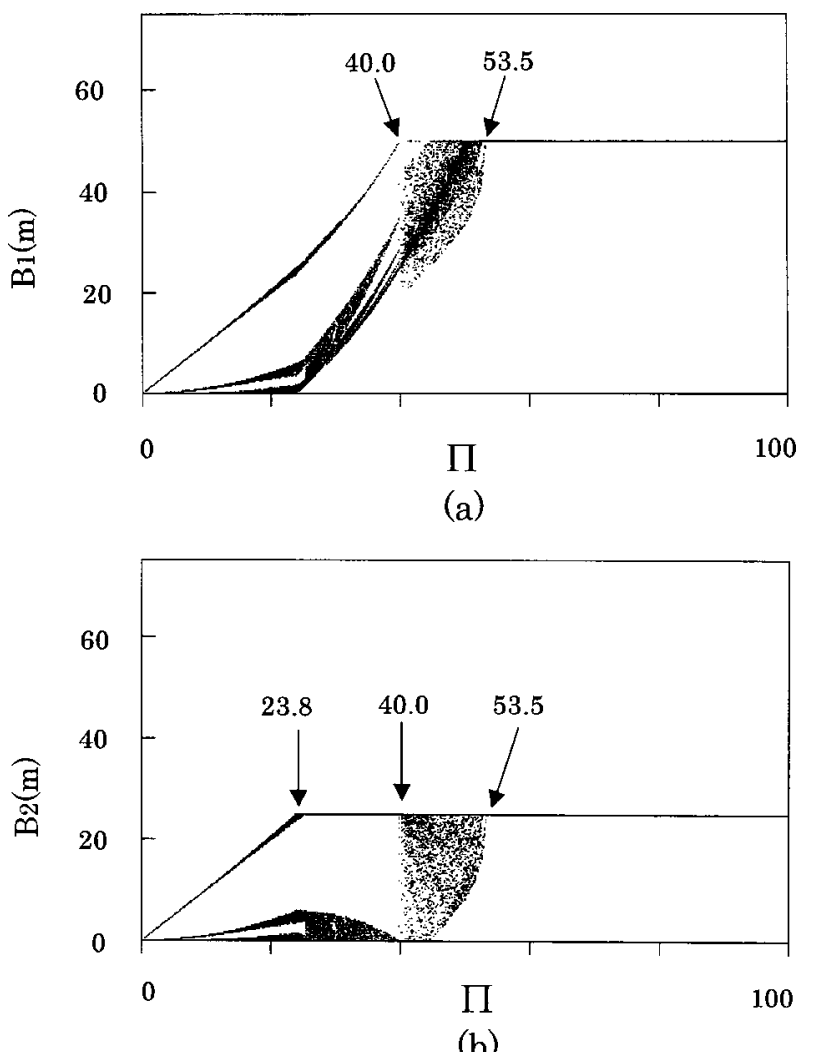

FIG. 7. (a) Plot of number $B_{1}(m)$ of riding passengers on bus 1 against loading parameter $\Pi$ from sufficiently large trip number $m$ $=900$ to 1000 where $M=2, \Gamma=0.01, F_{1, \max }=50, F_{2, \max }=25, V_{1}$ $=V_{2}=V_{0}$, and $a=0.0$. (b) Plot of the number $B_{2}(m)$ of riding passengers on bus 2 against loading parameter $\Pi$. 


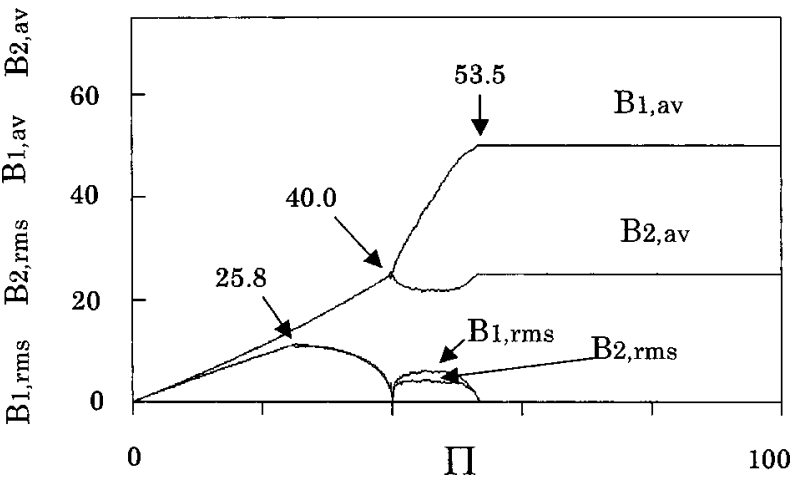

FIG. 8. Plots of means $B_{1, \text { av }}$ and $B_{2, \text { av }}$ and root mean squares $B_{1, \text { rms }}$ and $B_{2 \text {,rms }}$ of riding passenger numbers on buses 1 and 2 against loading parameter $\Pi$ where $M=2, \Gamma=0.01, F_{1, \max }=50$, $F_{2, \max }=25, V_{1}=V_{2}=V_{0}$, and $a=0.0$.

$=900$ to 1000 where $M=2, \Gamma=0.01, F_{1, \max }=50, F_{2 \text {,max }}$ $=25, V_{1}=V_{2}=V_{0}$, and $a=0.0$. Figure 7(b) shows a plot of the number $B_{2}(m)$ of riding passengers on bus 2 against the loading parameter $\Pi$. Figure 8 shows plots of the means $B_{1 \text {,av }}$ and $B_{2 \text {,av }}$ and root mean squares $B_{1, \mathrm{rms}}$ and $B_{2, \mathrm{rms}}$ of riding passenger numbers on buses 1 and 2 against the loading parameter $\Pi$. The mean and root mean square values for bus

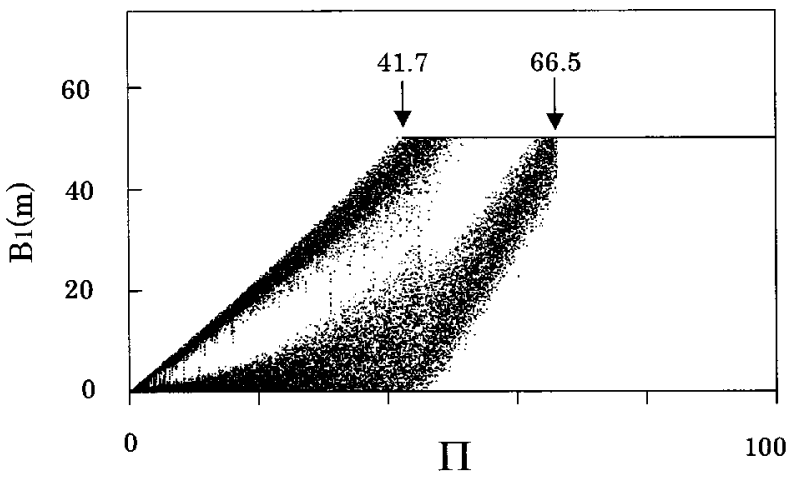

(a)

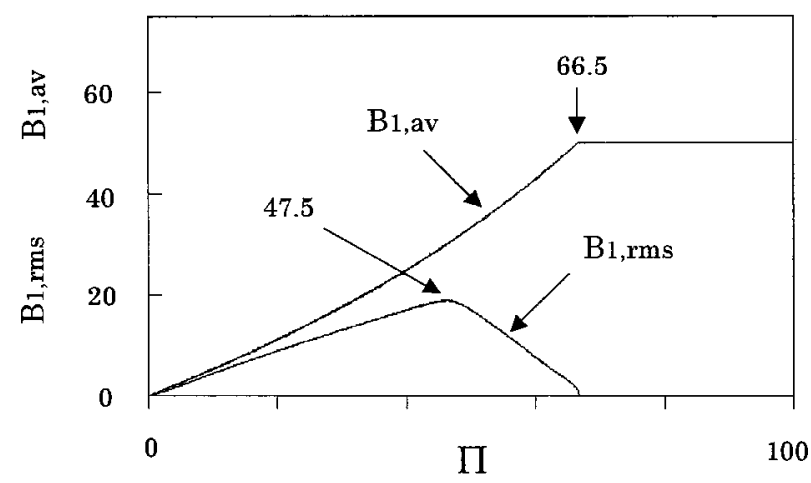

(b)

FIG. 9. Effect of noise on the number of riding passengers. (a) Plot of the number $B_{1}(m)$ of riding passengers against loading parameter $\Pi$ from sufficiently large trip number $m=900$ to 1000 for $a=10$, where $M=2, \Gamma=0.01$, and $F_{1, \max }=F_{2, \max }=50$. (b) Plots of mean $B_{1 \text {,av }}$ and root mean square $B_{1 \text {,rms }}$ of riding passenger numbers against loading parameter $\Pi$.

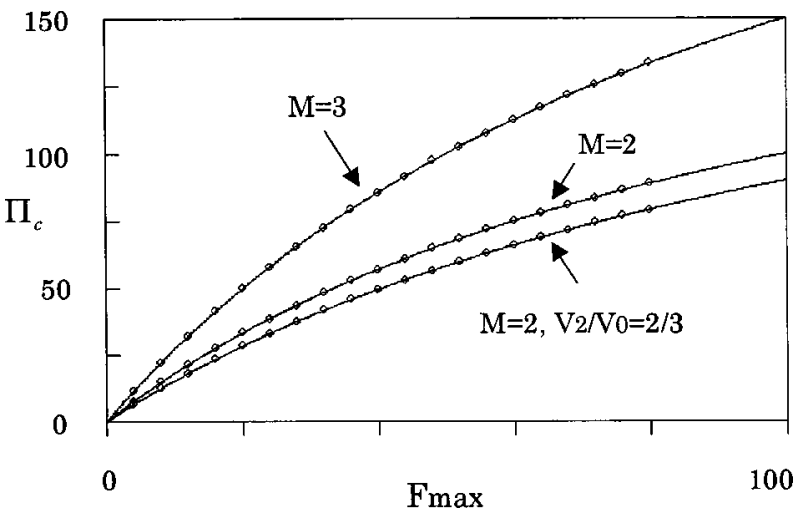

FIG. 10. Plots of threshold $\Pi_{c}$ against capacity $F_{\max }$ for $M$ $=2\left(V_{1}=V_{2}=V_{0}\right), M=2\left(V_{1}=V_{0}, V_{2}=2 V_{0} / 3\right)$, and $M=3\left(V_{1}\right.$ $\left.=V_{2}=V_{0}\right)$ where $\Gamma=0.01$. The solid curves represent the theoretical result (9). The circles indicate the simulation results.

1 are consistent with those of bus 2 until $\Pi=40$, and both mean values increase monotonically with loading parameter until $\Pi=40$. The root mean square values increase with loading parameter, reach their highest value at $\Pi=25.8$, become zero at $\Pi=40$, increase again, and become zero again at $\Pi_{c}=53.5$. When the loading parameter is higher than $\Pi_{c}=53.5$, both buses carry a full load of passengers. When the loading parameter is higher than 23.8 , bus 2 begins sometimes carrying the full load of passengers. When the loading parameter is higher than 40.0 , bus 1 begins sometimes carrying the full load of passengers. Thus, the number of riding passengers exhibits complex behavior due to the difference between the capacities of buses 1 and 2 .

\section{Noise effects}

We study the effect of noise on the bus motion. Figure 9(a) shows a plot of the number $B_{1}(m)$ of riding passengers against the loading parameter $\Pi$ from sufficiently large trip number $m=900$ to 1000 for $a=10$, where $M=2, \Gamma=0.01$, and $F_{1, \text { max }}=F_{2, \text { max }}=50$. Figure 9(a) is compared to Fig. 3(a), with no noise. The number of riding passengers changes its extended distribution. Figure 9(b) shows plots of the mean $B_{1 \text {,av }}$ and root mean square $B_{1, \text { rms }}$ of the riding passenger numbers against the loading parameter $\Pi$. The mean and rms of the riding passenger numbers change little. Thus, the noise does not affect the mean behavior of buses but has an effect on the distribution of the number of riding passengers. Therefore, the fluctuation of riding passenger numbers is attributable to the chaotic motion of buses induced by the interaction between buses and passengers.

\section{ANALYTICAL RESULTS}

We study analytically the dependence of the threshold (transition point) on the bus capacity. We consider a simple mass balance for the riding passengers. When the loading parameter is higher than the threshold $\Pi_{c}$, all buses carry a full load of passengers. So the following equation is obtained from Eq. (4): 


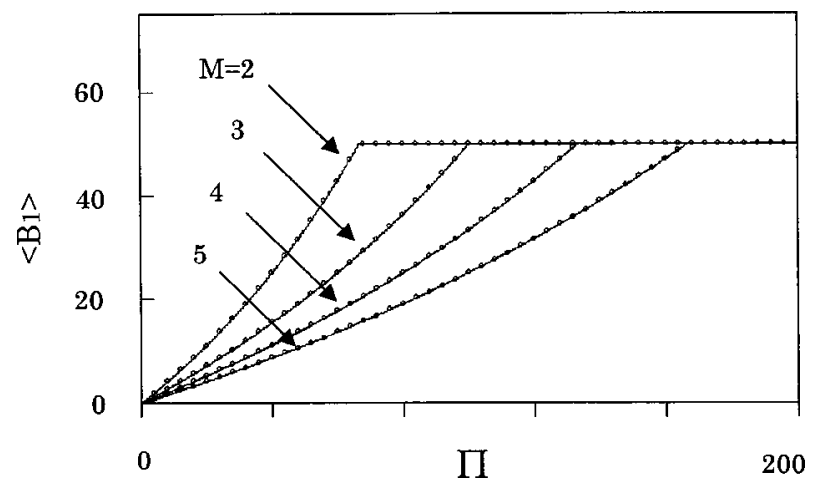

FIG. 11. Plots of mean number of riding passengers against loading parameter $\Pi$ for bus number $M=2-5$ where $\Gamma=0.01$, $F_{\max }=50$, and $a=0.0$. The solid curves represent the mean-field approximation (12). The circles indicate the simulation results.

$$
\Delta T_{i, c}=\Gamma F_{i, \max }+\frac{V_{0}}{V_{i}},
$$

where $\Delta T_{i, c}$ is the recurrence time (one period) of bus $i$ at the threshold. The number of passengers arriving at the origin over a sufficiently large time $T_{L}$ is given by $\Pi T_{L}$. At the threshold, the number $\Pi T_{L}$ of passengers is consistent with the number carried by all the buses. The following relationship holds:

$$
\sum_{i=1}^{M} \frac{T_{L}}{\Delta T_{i, c}} F_{i, \max }=\Pi_{c} T_{L} .
$$

One obtains the threshold from Eqs. (7) and (8):

$$
\Pi_{c}=\sum_{i=1}^{M} \frac{F_{i, \max }}{\Gamma F_{i, \max }+V_{0} / V_{i}} .
$$

Figure 10 shows plots of the threshold $\Pi_{c}$ against the capacity $F_{\max }$ for $M=2 \quad\left(V_{1}=V_{2}=V_{0}\right), \quad M=2\left(V_{1}=V_{0}, V_{2}\right.$ $\left.=2 V_{0} / 3\right)$, and $M=3\left(V_{1}=V_{2}=V_{0}\right)$ where $\Gamma=0.01$. The solid curves represent the theoretical result (9). The circles indicate the simulation results. The theoretical result (9) agrees with the simulation results.

We present the analytical result for the case where all the buses have the same speed $V_{i}=V_{0}$ and the same capacity $F_{i, \max }=F_{\text {max }}$. The mean recurrence time is given from Eq. (4) as

$$
\left\langle\Delta T_{i}\right\rangle=\Gamma\left\langle B_{i}\right\rangle+\frac{V_{0}}{V_{i}} .
$$

The number of passengers arriving at the origin over a sufficiently large time $T_{L}$ is given by $\Pi T_{L}$. Below the threshold, the number $\Pi T_{L}$ of passengers is consistent with those carried by all the buses. The following relationship holds:

$$
\sum_{i=1}^{M} T_{L} \frac{\left\langle B_{i}\right\rangle}{\left\langle\Delta T_{i}\right\rangle}=\Pi T_{L} .
$$

One obtains an approximation of riding passenger number as

$$
\left\langle B_{1}\right\rangle=\left\{\begin{array}{cc}
\frac{\Pi}{M-\Gamma \Pi} & \text { for } \Pi<\Pi_{c}, \\
F_{\max } & \text { for } \Pi \geqslant \Pi_{c} .
\end{array}\right.
$$

Figure 11 shows plots of the mean number of riding passengers against the loading parameter $\Pi$ for bus number $M$ $=2-5$ where $\Gamma=0.01, F_{\max }=50$, and $a=0.0$. The solid curves represent the approximation (12). The circles indicate the simulation results. The simulation results are consistent with the theoretical result.

\section{SUMMARY}

We have presented a dynamical model of shuttle buses that takes into account the interactions between buses and the maximal capacity of buses. The dynamics of the buses is expressed in terms of a coupled nonlinear map with noise. We studied the fluctuation of the number of riding passengers in a few shuttle buses that pass each other freely. We showed that the number of passengers carried by a bus exhibits a complex behavior with varying bus trips and the behavior of the buses exhibits deterministic chaos even if there is no noise. The fluctuation of the number of riding passengers is attributable to deterministic chaos. We showed that the chaotic motion depends on the loading parameter, the maximum capacity of the bus, the bus's speed, and the number of buses. We found that, when the loading parameter is higher than a threshold value, the dynamical transition from chaotic to periodic motion occurs because each bus carries a full load of passengers. We clarified the dependence of the threshold (transition point) on the capacity and speed of the buses. We presented an analytical result for a shuttle bus system. We showed that the analytical result is consistent with the simulation results.

The nonlinear map model of the buses is closely connected to dynamical models of elevators [29]. It is important to decide the capacity of the buses, the bus number, and estimate a passenger's waiting time in the bus operation system. The model presented here will be useful for bus system design.
[1] T. Nagatani, Rep. Prog. Phys. 65, 1331 (2002).

[2] D. Helbing, Rev. Mod. Phys. 73, 1067 (2001).

[3] D. Chowdhury, L. Santen, and A. Schadscheider, Phys. Rep. 329, 199 (2000).

[4] B. S. Kerner, Netw. Spatial Econ. 1, 35 (2001).

[5] Traffic and Granular Flow '99, edited by D. Helbing, H. J.
Herrmann, M. Schreckenberg, and D. E. Wolf (Springer, Heidelberg, 2000).

[6] K. Nagel and M. Schreckenberg, J. Phys. I 2, 2221 (1992).

[7] E. Ben-Naim, P. L. Krapivsky, and S. Redner, Phys. Rev. E 50, 822 (1994).

[8] E. Tomer, L. Safonov, and S. Havlin, Phys. Rev. Lett. 84, 382 
(2000)

[9] M. Treiber, A. Hennecke, and D. Helbing, Phys. Rev. E 62, 1805 (2000).

[10] H. K. Lee, H.-W. Lee, and D. Kim, Phys. Rev. E 64, 056126 (2001).

[11] I. Lubashevsky, S. Kalenkov, and R. Mahnke, Phys. Rev. E 65, 036140 (2002).

[12] I. Lubashevsky, R. Mahnke, P. Wagner, and S. Kalenkov, Phys. Rev. E 66, 016117 (2002).

[13] A. Kirchner and A. Schadschneider, Physica A 312, 260 (2002).

[14] K. Nishinari, D. Chowdhury, and A. Schadschneider, Phys. Rev. E 67, 036120 (2002).

[15] F. Weifeng, Y. Lizhong, and F. Weicheng, Physica A 321, 633 (2003).

[16] S. Maniccam, Physica A 321, 653 (2003).
[17] O. J. O’Loan, M. R. Evans, and M. E. Cates, Phys. Rev. E 58, 1404 (1998).

[18] D. Chowdhury and R. C. Desai, Eur. Phys. J. B 15, 375 (2000).

[19] T. Nagatani, Phys. Rev. E 63, 036116 (2001).

[20] H. J. C. Huijberts, Physica A 308, 489 (2002).

[21] S. A. Hill, e-print cond-mat/0206008.

[22] T. Nagatani, Physica A 297, 260 (2001).

[23] T. Nagatani, Phys. Rev. E 66, 046103 (2002).

[24] T. Nagatani, Phys. Rev. E 60, 1535 (1999).

[25] L. A. Safonov, E. Tomer, V. V. Strygin, Y. Ashkenazy, and S. Havlin, Chaos 12, 1006 (2002).

[26] G. F. Newell, Transp. Res., Part B: Methodol. 32, 583 (1998).

[27] T. Nagatani, Physica A 319, 568 (2002).

[28] T. Nagatani, Physica A 323, 686 (2003).

[29] T. Poschel and J. Gallas, Phys. Rev. E 50, 2654 (1994). 